Abstract ID: 20

\title{
Osteochondral defect repair via autologous implantation of three dimensional construct engineered from poly (lactic- co-glycolic acid) / fibrin hybrid scaffold seeded with bone marrow mesenchymal stem cells
}

Rozlin Abdul Rahman ${ }^{\mathrm{a}}$ | Norhamiza Mohamad Sukri ${ }^{\mathrm{a}}$ | Noorhidayah Md Nazir ${ }^{\mathrm{a}}$ | Muhammad Aa'zamuddin Ahmad Radzi ${ }^{\mathrm{a}}$ | Ahmad Hafiz Zulkifly ${ }^{\mathrm{b}}$ | Munirah Sha'ban ${ }^{\mathrm{a}}$

${ }^{a}$ Kulliyyah of Allied Health Sciences, International Islamic University Malaysia

${ }^{b}$ Kulliyyah of Medicine, International Islamic University Malaysia

Introduction: Treating damaged articular cartilage is a predicament in the orthopaedic field due to its limited capacity for regeneration. Efforts to find the best available treatment for articular cartilage injuries are still actively ongoing. This study investigates the ability of the autologous implantation approach using three dimensional (3D) constructs engineered from bone marrow mesenchymal stem cells (BMSCs) seeded on poly(lactic-co-glycolic acid) (PLGA) with or without fibrin as cells carrier for the repair of full-thickness osteochondral defect in rabbit model. Methods: 3D PLGA was fabricated via solvent casting-salt leaching technique using salt as porogen. The BMSCs obtained from the New Zealand white rabbits were cultured and mixed with (1) autologous plasma-derived fibrin or, (2) culture media which served as the control, prior to 'cells seeding' procedure into the PLGA. After three weeks of in vitro culture, the engineered 3D constructs PLGA/Fibrin/BMSCs (PFB) and PLGA/BMSCs (PB) - were implanted autologously to the osteochondral defect created in the rabbit's knee. The in vivo constructs were harvested en bloc and evaluated by gross inspection and histology, gene expression, sulphated glycosaminoglycan (sGAG) production and biomechanical defect property at 6 and 12 weeks post implantation ( $\mathrm{n}=6$ for each group). Results: The results showed that the osteochondral defects treated with the PFB constructs exhibited better repairment, more cartilaginous extracellular matrix, higher sGAG content, significantly stronger compressive strength and greater expression of chondrogenic marker genes than the PB group. Conclusions: The findings suggest that PFB with optimal induction are feasible to treat osteochondral and may become a potential treatment modality aiming to treat articular cartilage disease in human.

KEYWORDS: cartilage tissue engineering, poly(lactic-co-glycolic acid), bone marrow mesenchymal stem cells, fibrin, scaffold 